\title{
EMPTY NAMES AND NEGATIVE EXISTENTIALS
}

\author{
Ricardo Mena \\ Universidad Nacional Autónoma de México \\ Instituto de Investigaciones Filosóficas \\ ricardo.mena21@gmail.com
}

This Special Issue concerns two interrelated problems: the problem of empty names and the problem of singular negative existentials. These problems are particularly pressing for Millians: roughly, those who think that the meaning of a name is just its referent, or, alternatively, that the contribution that a name makes to a proposition is its referent. Let's start with the problem of empty names. An empty name is a name that lacks a referent. Suppose that the name "Nina" is empty. Furthermore, suppose that for whatever reason we mistakenly believe that "Nina" refers to a cat. Intuitively, if I say "Nina has yellow eyes", I have said something meaningful: for sure you can understand what I said. However, this is hard to explain if the meaning of "Nina" is exhausted by its referent and there is no such thing. ${ }^{1}$ The problem gets a bit sharper when we consider fictional names. Intuitively, if I say "Frodo is an adventurous hobbit", I have said something both meaningful and true. It's already hard to explain why that sentence is meaningful, supposing that "Frodo" is empty: now we also have to explain why that sentence is (or at least seems to be) true. Given standard compositional semantics, a sentence of that form is true only if the referent is in the extension of the predicate. If "Frodo" is empty, it is far from obvious that "Frodo is an adventurous hobbit" can be true. This leg of the problem is had by Millians and non-Millians alike.

The problem of singular negative existentials and the problem of empty names aren't completely orthogonal with respect to each other. If you ever feel inclined to assert a sentence like "Celeste doesn't exist", it's quite likely that you think that "Celeste" is an empty name. Let's say that "Celeste" is in fact empty. Then, intuitively, what you said is true. But how can this be, if you are not talking about anything? So there is the problem of explaining what in the world

\footnotetext{
${ }^{1}$ Notice that a Descriptivist doesn't have this problem.
} 
could make that sentence true. If I say "Judith Polgar is a great chess player" it's fairly clear which parcel of the world makes that sentence true, especially because Judith Polgar does exist. However, it is far less clear which parcel of the world, if any, could be responsible for the truth of "Celeste doesn't exist". That is the metaphysical aspect of the problem. Of course, there are semantic aspects as well. If "Celeste" is empty, how could the compositional semantic mechanisms work to determine the truth-value of the negative existential? Again, given standard compositional semantics, if a singular atomic sentence is true, it has to be the case that the referent of the singular term is in the extension of the predicate. If "Celeste" is empty, there is a problem in holding that "Celeste doesn't exist" is true. Of course, there is the further problem of explaining how singular negative existentials can be meaningful, especially if one is a Millian.

This Special Issue contains five papers dealing with these problems. Dickie's and Fontaine's papers tackle the problem of empty names. Yablo, Clapp, and De Rizzo's papers tackle the problem of singular negative existentials. In what follows I'll offer a brief presentation of each of the papers.

Imogen Dickie

(Invited Submission)

This paper assigns a novel place to the study of empty singular terms. Rather than having our theory of empty singular terms drop out of our theory of belief formation and reference, Dickie proposes to have our theory of belief formation and reference drop out of our theory of empty names - or at the very least she shows that a strong line of argument can proceed that way. Dickie argues that a proper understanding of empty singular terms can be used to argue in favour of a lemma concerning ordinary belief forming activities. This, in turn, can be used to argue in favour of a particular theory of reference. The lemma in question is this: "Part of the aim of ordinary belief-forming activity is to secure and maintain aboutness relations with particular things."

Two emptiness cases get special attention: accidental emptiness and fiction. Cases of accidental emptiness are ones where we use empty singular terms (and we form beliefs involving them) without knowing that the terms in question are empty. Dickie's insight here is to understand what goes wrong in these cases in order to understand instances where belief formation and reference proceed smoothly. The upshot of Dickie's discussion is that the aboutness of 
the beliefs we form in accidental emptiness cases is not required for attempting to form those beliefs, it is only required for the attempt to succeed. This, plus other considerations, strongly suggest that the aim of belief-forming activity is to maintain the relevant aboutness relations, as the lemma states. According to Dickie, engaging with fiction involves genuine pretence. Within the scope of this pretence, we treat the fiction as if it was real, using the text as a source of testimony on the basis of which we sustain aboutness relations with individuals. If this is what goes on in the case of fiction, then understanding what goes on with uptake in real cases is just a matter of tossing away pretence. This, again, constitutes a strong argument in support for the lemma.

\section{Matthieu Fontaine}

According to the Artifactual Theory of Fiction, fictional names refer to a certain kind of abstract entity: artefacts created by authors. These abstract entities are ontologically dependent on their author and fiction. Thus, in this view, fictional names aren't empty: their referents are of a special kind. This paper tackles a set of related problems that this theory faces. For instance, this theory has to account for the possibility of cross-fictional reference: when a name in a given fiction refers to a fictional character from another fiction. I could, for example, write a short story and, in it, I could refer to Doyle's Sherlock Holmes by way of using the name "Sherlock". Another problematic case is one where two different characters from different fictions merge into a single character. Furthermore, it could happen that a single character splits into two different characters. It is easy to see how a straightforward theory of rigid designation would have serious problems accounting for these cases.

Fontaine proposes to solve these problems by appealing to something like Hintikka's notion of individuals as world lines. Individuals should be distinguished from objects that are part of particular possible worlds: these objects are manifestations of individuals in those worlds, but they are not individuals. Objects in different possible worlds can be linked by a world line, and these world lines are individuals. Of course, two different world lines can merge, by being linked to the same object at a particular world. A single world line can also split, creating two different world lines, linked to two different objects at a particular world. These world lines are created by agents and their intentions. Fontaine argues that it is plausible to think that fictional characters are world lines, created by fiction 
writers. In the paper he explains with all detail how this way of thinking about things solves the problems had by the Artefactual Theory of Fiction. This paper also tackles interesting issues concerning quantification over fictional characters understood this way.

\section{Stephen Yablo \\ (Invited Submission)}

In Yablo's view "Holmes doesn't exist" is true, because "Holmes exists" is false. Why is the latter sentence false? Because it misdescribes the things that exist: it claims that Holmes is among them. For this to be the case, we need to interpret "Holmes exists" as a description of the things that exist. This can easily be achieved in conversations where the topic is something like the things that exist: so there is a bit of context sensitivity going on here. For "Holmes exists" to be true, interpreted this way, it has to be the case that there is an $\mathrm{x}$ such that, $\mathrm{x}=$ Holmes is true. But there is no such $\mathrm{x}$. How could something satisfy $\lambda \mathrm{x}(\mathrm{x}=$ Holmes $)$, if "Holmes" is empty? So "Holmes exists" is false, qua description of the things that exist. Now the problem is to explain how "Sherlock Holmes doesn't exist" and "Anna Karenina doesn't exist" differ in cognitive value if both names are empty. After all, one may think that nothing satisfies- $\lambda \mathrm{x}(\mathrm{x}=$ Karenina $)$ for the same reasons nothing satisfies $-\lambda \mathrm{x}$ $(\mathrm{x}=$ Holmes): nothing can satisfy $\lambda \mathrm{x}(\mathrm{x}=a)$, if " $a$ " is empty. This, however, is not a problem for Yablo, because, according to him, "[t]hat a name's meaning is exhausted by its referent does not mean that the name's truth-conditional contribution is exhausted by its referent". This is particularly so when names appear in indicative conditionals. In Yablo's view, what "Holmes doesn't exist" conveys is that everything is: not Holmes, even if Holmes exists. On the other hand, "Karenina doesn't exist" conveys that everything is: not Karenina, even if Karenina exists. It is treated as a datum that these two indicative conditionals express different conditions, even if both names are empty.

\section{Lenny Clapp}

In his paper Clapp tackles what he calls the referential problem of negative existentials: speakers sometimes utter sentences such as 'Sherlock Holmes does not exist' and at least purport to refer to something and predicate 'does not exist' of it. But this seems to be incoherent: if, as Quine famously maintained, everything exists, 
then it makes no sense to assert of something that it does not exist. The problem is that, despite this seeming incoherence, we typically interpret such speakers as asserting something not only coherent, but even true. Clapp calls his solution to this problem pragmatic Meinongianism. The essential idea is that sometimes the predicate 'does not exist' is pragmatically modulated to express an inclusive sense, a sense which allows that the predicate may be satisfied by certain sorts of things - e.g. fictional characters.

To motivate pragmatic Meinongianism, Clapp first criticizes Salmon's 1998 Russell-inspired solution to the referential sub-problem, and then responds to the charge that appeal to the pragmatic modulation of 'does not exist' is ad hoc. To meet this charge, Clapp notes that pairs such as

(la) Sherlock Holmes doesn't really exist; he is just a fictional character.

(lb) Sherlock Holmes doesn't exist; he's a fictional character.

and

(2a) Laura doesn't really run; she's just a jogger.

(2b) Laura doesn't run; she's a jogger.

give rise to analogous puzzling phenomena: just as a use of (lb) would seem to be incoherent because being a fictional character seems to require existing, so a use of $(2 \mathrm{~b})$ would seem to be incoherent because being a jogger seems to require running. Clapp argues that the solution to the puzzle in the case of $(2 \mathrm{~b})$ is that sometimes the unmodified predicate 'doesn't run' is pragmatically modulated to mean what is more explicitly meant by the modified predicate 'doesn't really run' in (2a), and moreover what is explicitly meant by this modified predicate is an inclusive sense; the result of this pragmatic modulation is that some things that satisfy 'doesn't run' also satisfy 'is a jogger'. Given the obvious analogies between the pairs, an analogous explanation should be applied to the puzzle raised by uses of (lb): sometimes the unmodified predicate 'doesn't exist' is pragmatically modulated to mean what is more explicitly meant by the modified predicate 'doesn't really exist' in (la), and moreover what is explicitly meant by this modified predicate is an inclusive sense; the result of this pragmatic modulation is that some things that satisfy 'doesn't exist' also satisfy 'is a fictional character'. The 
upshot is that pragmatic modulation is required to explain analogous, yet independent, puzzles, so it is not ad hoc to appeal to pragmatic modulation to solve the referential problem of negative existentials.

Finally, Clapp concludes by arguing that, despite what one might suppose, pragmatic Meinongianism does not imply fictional realism, nor any other ontologically profligate view. Clapp suggests that this ontological neutrality is a further virtue of his view.

\section{Julio De Rizzo}

In this paper De Rizzo criticizes a natural proposal for the grounds of negative existential truths, and then offers an alternative theory that doesn't suffer from the same difficulties. According to the natural proposal that De Rizzo considers, the truth of "Vulcan does not exist" is grounded in the following totality truth: this exists, that exists, ..., that exists, and nothing else exists. This proposal is attractive because, according to it, the truth of negative existentials is grounded in things that exist (in the way the world is), and not in things that do not exist. However, De Rizzo argues that, even though it is natural, this proposal suffers from three serious shortcomings. Here I'll only consider one of them. Roughly, the problem is that a ground, even if only partial, must be explanatorily relevant. Thus, the existence of Quine partially grounds the existence of $\{$ Quine, Socrates\}, and it is also explanatorily relevant regarding the existence of the latter set. Then, since the totality truth is partially grounded in the existence of my cat, and partial grounding is transitive, it seems that according to the natural proposal the existence of my cat partially grounds the truth of "Vulcan doesn't exist" and, as such, it should be explanatory relevant for the truth of that negative existential. However, that is absurd: my cat's existence is not explanatorily relevant for the truth of "Vulcan doesn't exist". De Rizzo puts forward two other objections with a similar flavor.

As a way of avoiding these problems, De Rizzo proposes that the proper theory should find the grounds for all negative existential truths at once. So, rather than arguing that the grounds for negative existential $\Phi$ is such and such, and the ground for negative existential $\psi$ is such and such, we should take $\Gamma$, the collection of all negative existentials, and argue that its ground is the same totality truth as before: this exists, that exists, ..., that exists, and nothing else exists. Thus, in this view, the grounding relation is many-many, and not many-one. De Rizzo argues that the proper version of the relevance 
constraint for a many-many grounding relation holds irreducibly between pluralities. Hence, "if $\Gamma$ because $\Delta$, then $\Delta$ is relevant to $\Gamma$, even if, were the truths therein taken separately, relevance is not 'distributed' between these pluralities". This solves the relevance problem, since my cat's existence, considered in isolation, shouldn't be considered when it comes to relevance concerning many-many grounding claims. 